Pacific Journal of Mathematics

RATES OF DECREASE OF SEQUENCES OF POWERS IN 


\section{RATES OF DECREASE OF SEQUENCES OF POWERS IN COMMUTATIVE RADICAL BANACH ALGEBRAS}

\section{JEAN Esterle}

Every element $b$ of a radical Banach algebra $\mathscr{R}$ satisfies $\lim _{n \rightarrow \infty}\left\|b^{n}\right\|^{1 / n}=0$. We are concerned here with the existence of a lower bound for the rate of decrease of the sequence $\left(\left\|b^{n}\right\|\right)$ under various assumptions over $b$ and $\mathscr{R}$, when $b$ is not nilpotent and $\mathscr{R}$ commutative.

If the nilpotents are dense in $\mathscr{R}$ then for every sequence $\left(\lambda_{n}\right)$ of positive reals there exists a nonnilpotent $b \in \mathscr{R}$ such that $\lim \inf _{n \rightarrow \infty}\left\|b^{n}\right\| / \lambda_{n}=0$. A stronger result holds if $\mathscr{R}$ possesses furthermore a bounded approximate identity. On the other direction if $\mathscr{R}$ has no nilpotent element and if some element of $\mathscr{R}$ which is not a divisor of zero acts compactly on $\mathscr{R}$ then there exists a sequence $\left(\lambda_{n}\right)$ of positive reals such that $\liminf _{n \rightarrow \infty}\left\|b^{n}\right\| / \lambda_{n}=+\infty$ for every nonzero $b \in \mathscr{R}$. Also there exists universal lower bounds for the rate of decrease of $\left\|a^{n}\right\|$ if $\left(a^{t}\right)$ is an analytic semigroup over the positive reals or over some open angle. Such lower bounds do not exist for infinitely differentiable semigroups over the positive reals.

1. W. G. Badé and H. G. Dales observed recently in [3] that given any sequence $\left(\lambda_{n}\right)$ of positive reals there exists a nonnilpotent element $b$ of the Volterra algebra $L_{*}^{1}(0,1)$ such that $\left\|b^{n}\right\|<\lambda_{n}$ for every $n \in N$.

On the other hand they showed in [3] that if $\omega$ is a continuous positive function over $[0,+\infty[$ satisfying

$$
\lim _{x \rightarrow \infty}[\omega(x)]^{1 / x}=0, \quad \omega(x+y) \leqq \omega(x) \omega(y)
$$

for every $x, y \in\left[0,+\infty\left[\right.\right.$ then there exists a sequence $\lambda_{n}(\omega)$ of positive reals such that

$$
\liminf _{n \rightarrow \infty} \frac{\left\|b^{n}\right\|}{\lambda_{n}(\omega)}=+\infty
$$

for every nonzero elements $b$ of $L^{1}\left(R^{+}, \omega\right)$. Precise estimations of this sequence $\lambda_{n}(\omega)$ are given in [3] (also Badé and Dales obtain in [3] a characterization of the "standard" closed ideals of $L^{1}\left(R^{+}, \omega\right)$ in terms of rates of decrease of the sequence of powers of their elements).

We first study in $\S 2$ commutative radical Banach algebras which possess nilpotent elements. The Theorem 2.1 shows that if $b \in[b \mathscr{N}]^{-}$ for some nonnilpotent element $b$ of a commutative algebra $\mathscr{R}$, where 
$\mathscr{N}$ is the nilradical of $\mathscr{R}$, then for every sequence $\left(\lambda_{n}\right)$ of positive reals there exists a nonnilpotent element $c$ of $\mathscr{R}$ such that

$$
\liminf _{n \rightarrow \infty} \frac{\left\|c^{n}\right\|}{\lambda_{n}}=0, b \in c[\mathscr{R} \oplus C e]^{-} .
$$

We obtain a stronger result when $\mathscr{R}$ possesses bounded approximate identities: if the nilpotents are dense in such an algebra $\mathscr{R}$ and if $\mathscr{R}$ is separable then for every sequence $\left(\lambda_{n}\right)$ of positive reals there exists $b \in \mathscr{R}$ such that $[b \mathscr{R}]^{-}=\mathscr{R}$ and such that $\left\|b^{n}\right\|<\lambda_{n}$ for every $n \in N$. Using results recently obtained by the author in [9] we incidentally deduce that if the continuum hypothesis is satisfied then for every commutative complex algebra $A$ without unit which is an integral domain of cardinality $2^{\aleph_{j}}$ and for every sequence $\left(\lambda_{n}\right)$ of positive reals there exists a faithful algebra homomorphism $\varphi: A \rightarrow \mathscr{R}$ such that $\lim \sup _{n \rightarrow \infty}\left\|\varphi\left(a^{n}\right)\right\| / \lambda_{n}=0$ for every nonzero $a \in A$.

In $\S 3$ we study the behavior of $\left\|a^{z}\right\|$ where $\left(a^{z}\right)$ is a (nonzero) analytic semigroup in a commutative radical Banach algebra $\mathscr{R}$ defined over an open angle $U_{\theta}=\{z \in C-\{0\}|| \operatorname{Arg} z \mid<\theta\}$ where $0<$ $\theta \leqq \pi / 2$. (A.M. Sinclair proved in [12] that if $\mathscr{R}$ possesses bounded approximate identities then $\mathscr{R}$ contains analytic semigroups $\left(a^{z}\right)$ defined over $U_{\pi / 2}$ which are bounded over $] 0,+\infty[$.$) We prove that$ for such an analytic semigroup we have $\lim \log _{r \rightarrow \infty}\left\|a^{r e^{i} \varphi}\right\| / r^{r}=0$ for every $\gamma>\pi / 2 \theta$ and for every $\varphi \in]-\theta, \theta[$, the convergence being uniform over $[-\alpha, \alpha]$ for every $\alpha \in[0, \theta[$. (This result is related with the Ahlfors-Heins theorem for continuous bounded functions over the closed right-hand plane which are analytic over the open right-hand halfplane). In fact these estimates work for any continuous algebra seminorm $q$ over $\mathscr{R}$ such that $q(a) \neq 0$. In particular, the notations being as above, we have lim $\inf _{n \rightarrow \infty}\left[q\left(a^{n}\right)\right]^{1 / n} / \exp \left[-n^{\gamma-1}\right]=+\infty$ for every $\gamma>\pi / 2 \theta$ if $q$ is a continuous algebra seminorm over $\mathscr{R}$ such that $q(a) \neq 0$. We also obtain lower estimates for the rate of decreasing at infinity of the function $r \rightarrow\left\|a^{r}\right\|$ where $\left(a^{r}\right)_{r>0}$ is a (nonzero) analytic semigroup in $\mathscr{R}$ defined over $] 0,+\infty[$. Such lower estimates do not exist for infinitely differentiable semigroups $\left(a^{r}\right)_{r>0}$ over ]0, $+\infty[$. We show in the Theorem 3.6 that for every continuous positive function $g$ over $[1,+\infty$ [ there exists a commutative Banach algebra $\mathscr{R}$ and an infinitely differentiable semigroup $\left(a^{r}\right)_{r>0}$ in $\mathscr{R}$ defined over ]0, $+\infty$ [ such that $0<\left\|a^{r}\right\|<g(r)$ for every $r \geqq 1$.

In $\S 4$ we study the rate of decrease of sequences of powers in commutative radical Banach algebras which are integral domains and in which some nonzero element acts compactly. We prove that for every continuous algebra norm $q$ defined on such an algebra $\mathscr{R}$ there exists a sequence $\lambda_{n}(q)$ such that 


$$
\liminf _{n \rightarrow \infty} \frac{q\left(b^{n}\right)}{\lambda_{n}(q)}=+\infty
$$

for every nonzero element $b$ of $\mathscr{R}$. W. G. Badé and H. G. Dales showed in [3] that every element of $L^{1}\left(R^{+}, \omega\right)$ acts compactly if $\omega$ is a continuous weight over $[0, \infty$ [ such that $\omega(x+y) \leqq \omega(x) \omega(y)$ for every $x, y \geqq 0$ and

$$
\lim _{x \rightarrow \infty}[\omega(x)]^{1 / x}=0
$$

provided the function $x \rightarrow[\omega(x)]^{1 / x}$ is decreasing over $[0,+\infty[$. We thus obtain in a very simple way the existence of a lower bound for the rate of decrease of sequences of powers in the weighted algebras $L^{1}\left(R^{+}, \omega\right)$ for every weight $\omega$ which is continuous over ]0, $+\infty$ [ (even if the weight is unbounded at the origin), but of course we do not obtain a concrete estimate for this lower bound.

Note that G. R. Allan and A. M. Sinclair proved in [2] that if a commutative radical Banach algebra $\mathscr{R}$ has a bounded approximate identity then for any sequence $\left(\lambda_{n}\right)$ of positive reals such that $\lim _{n \rightarrow \infty} \lambda_{n}=0$ there exists $b \in \mathscr{R}$ such that $\lim \inf _{n \rightarrow \infty}\left\|b^{n}\right\|^{1 / n} / \lambda_{n}=+\infty$. In other terms the sequence $\left(\left\|b^{n}\right\|\right)$ may decrease as slowly as possible in this case. It was also recently proved by the author in [8] that the rate of decrease of the sequence $\left(\left\|b^{n}\right\|\right)$ is usually very irregular. If $b \in[b \mathscr{R}]^{-}$for some nonnilpotent element $b$ of a commutative radical Banach algebra $\mathscr{R}$ then there exists $c \in \mathscr{R}$ such that

$$
\liminf _{n \rightarrow \infty} \frac{\left\|c^{n}\right\|^{1 / n}}{\left\|b^{n}\right\|^{1 / n}}=0 ; \quad \limsup _{n \rightarrow \infty} \frac{\left\|c^{n}\right\|^{1 / n}}{\left\|b^{n}\right\|^{1 / n}}=+\infty .
$$

I must thank W. G. Badé and G. H. Dales for sending me preprints and Paul Koosis and A. M. Sinclair for several valuable discussions.

I thank also the referee who checked very carefully the original version of this paper.

2. Rate of decrease of sequences of powers in commutative radical Banach algebras which possess nilpotent elements.

THEOREM 2.1. Let $\mathscr{R}$ be a commutative radical Banach algebra and let $\mathscr{N}$ be the set of nilpotent elements of $\mathscr{R}$. If $b \in[b \mathscr{N}]^{-}$for some nonnilpotent element $b$ of $\mathscr{R}$ then for every sequence $\left(\lambda_{n}\right)$ of positive reals there exists a nonnilpotent element $c$ of $\mathscr{R}$ such that

$$
\liminf _{n \rightarrow \infty} \frac{\left\|c^{n}\right\|}{\lambda_{n}}=0
$$


Proof. Add a unit $e$ to $\mathscr{R}$. We will construct by induction a sequence $\left(e_{n}\right)$ of elements of $\mathscr{N}$, a sequence $\left(\mu_{n}\right)$ of positive reals and a strictly increasing sequence $\left(q_{n}\right)$ of positive integers satisfying the following conditions, where we denote by $X_{n}$ the product $b\left(\mu_{1} e+e_{1}\right), \cdots,\left(\mu_{n} e+e_{n}\right)$ (we put for convenience $X_{0}=b, \mu_{0}=1$, $\left.q_{0}=1\right)$.

(1) $\left\|X_{n-1}^{q_{k}}-X_{n}^{q_{k}}\right\|<2^{-n} \lambda_{q_{k}}$ for every $k<n$ and every $n \geqq 1$.

(2) $\left\|X_{n}^{q_{n}}\right\|<2^{-n} \lambda_{q_{n}}$ for every $n \geqq 1$.

$$
\begin{aligned}
\left\|X_{n-1}-X_{n}\right\|<2^{-n}\left(1+\left\|\left(\mu_{1} e+e_{1}\right)^{-1}\right\|\right)^{-1} \cdots & \\
& \cdots\left(1+\left\|\left(\mu_{n-1} e+e_{n-1}\right)^{-1}\right\|\right)^{-1} \quad \text { for every } n \geqq 1 .
\end{aligned}
$$

We can choose a nonzero element $e_{1}$ of $\mathscr{N}$ such that

$$
\left\|b-b e_{1}\right\|<1 / 2 \max \left(1, \lambda_{1}\right) .
$$

Let $q_{1}$ be some positive integer such that $e_{1}^{q_{1}}=0$. Then

$$
\lim _{\substack{\mu \rightarrow 0 \\ \mu>0}} b^{q_{1}}\left(e_{1}+\mu e\right)^{q_{1}}=0
$$

(use the Newton polynomial). So taking $\mu_{1}>0$ small enough we can arrange the conditions (1), (2), (3) to be satisfied for $n=1$. Now suppose that we have constructed a finite sequence $\left(e_{1}, \cdots, e_{n}\right)$ of elements of $\mathscr{N}$, a finite sequence $\left(\mu_{1}, \cdots, \mu_{n}\right)$ of positive reals and a finite increasing sequence $\left(q_{1}, \cdots, q_{n}\right)$ of positive integers satisfying the three conditions. As $b \in[b \mathscr{N}]^{-}$we have also $X_{n} \in\left[X_{n} \mathscr{N}\right]^{-}$and there exists a sequence $\left(f_{m}\right)$ of elements of $\mathscr{N}$ such that

$$
\lim _{m \rightarrow \infty}\left\|X_{n}-X_{n} f_{m}\right\|=0 \text {. }
$$

So

$$
\lim _{m \rightarrow \infty}\left\|X_{n}^{q_{k}}-X_{n}^{q_{k}} \cdot f_{m}^{q_{k}}\right\|=0
$$

for every $k \leqq n$ and taking $e_{n+1}=f_{m}$ for $m$ large enough, we can arrange that

$$
\left\|X_{n}^{q_{k}}-X_{n}^{q_{k}} e_{n+1}^{q_{k}}\right\|<2^{-(n+1)} \lambda_{q_{k}}
$$

for every $k \leqq n$ and

$$
\begin{gathered}
\left\|X_{n}-X_{n} e_{n+1}\right\|<2^{-n-1}\left[1+\left\|\left(\mu_{1} e+e_{1}\right)^{-1}\right\|\right]^{-1} \cdots \\
\cdots\left[1+\left\|\left(\mu_{n} e+e_{n}\right)^{-1}\right\|\right]^{-1} .
\end{gathered}
$$

Then

$$
\lim _{\mu \rightarrow 0} \sup \left\|X_{n}^{q_{k}}-X_{n}^{q_{k}}\left(e_{n+1}+\mu e\right)^{q_{k}}\right\|<2^{-n-1} \lambda_{q_{k}},
$$




$$
\begin{gathered}
\limsup _{\substack{\mu \rightarrow 0 \\
n>0}}\left\|X_{n}-X_{n}\left(e_{n+1}+\mu e\right)\right\|<2^{-n-1}\left[\left\|\left(\mu_{1} e+e_{1}\right)^{-1}\right\|+1\right]^{-1} \cdots \\
\cdots\left[\left\|\left(\mu_{n} e+e_{n}\right)^{-1}\right\|+1\right]^{-1} .
\end{gathered}
$$

Also if $q_{n+1}>q_{n}$ is some integer such that $e_{n+1}^{q_{n+1}}=0$,

$$
\lim _{\substack{n \rightarrow 0 \\ i>0}} \sup \left\|X_{n}^{q_{n+1}}\left(\mu e+e_{n+1}\right)^{q_{n+1}}\right\|=0,
$$

(use again the Newton polynomial).

So choosing $\mu_{n+1}>0$ small enough we can arrange the conditions (1), (2), (3) to be satisfied by the finite sequences $\left(e_{1}, \cdots, e_{n+1}\right),\left(q_{1}, \cdots, q_{n+1}\right)$ and $\left(\mu_{1}, \cdots, \mu_{n+1}\right)$. We thus see that we can construct by induction three infinite sequences $\left(e_{n}\right),\left(q_{n}\right)$ and $\left(\mu_{n}\right)$ satisfying the three conditions.

Using the condition (3) we see that the sequence $\left(X_{n}\right)$ is Cauchy. Denote by $c$ its limit. We have, for every $n \in N$ :

$$
\begin{aligned}
\left\|c^{q_{n}}\right\| & =\left\|X_{n}^{q_{n}}+\sum_{m=n}^{\infty}\left(X_{m+1}^{q_{n}}-X_{m}^{q_{n}}\right)\right\| \\
& \leqq\left\|X_{n}^{q_{n}}\right\|+\sum_{m=n+1}^{\infty}\left\|X_{m}^{q_{n}}-X_{m-1}^{q_{n}}\right\| .
\end{aligned}
$$

Using the conditions (1) and (2), we obtain:

$$
\left\|c^{q_{n}}\right\| \leqq 2^{-n+1} \lambda_{q_{n}} .
$$

So

$$
\liminf _{n \rightarrow \infty} \frac{\left\|c^{n}\right\|}{\lambda_{n}}=0
$$

Also

$$
\begin{gathered}
\left\|c\left(\mu_{1} e+e_{1}\right)^{-1} \cdots\left(\mu_{n} e+e_{n}\right)^{-1}-b\right\| \\
\leqq \| \sum_{m=n+1}^{\infty} X_{m}\left(\mu_{1} e+e_{1}\right)^{-1} \cdots\left(\mu_{n} e+e_{n}\right)^{-1}-X_{m-1}\left(\mu_{1} e+e_{1}\right)^{-1} \cdots \\
\cdots\left(\mu_{n} e+e_{n}\right)^{-1} \|
\end{gathered}
$$

Using (3), we obtain: $\left\|c\left(\mu_{1} e+e_{1}\right)^{-1} \cdots\left(\mu_{n} e+e_{n}\right)^{-1}-b\right\| \leqq 2^{-n}$. So $b \in$ $[c(\mathscr{R} \oplus \boldsymbol{C} e)]^{-}$, and $c$ is not nilpotent, which achieves the proof of the theorem.

We will now prove a stronger result for commutative radical Banach algebras which possess approximate identities.

TheOREM 2.2. Let $\mathscr{R}$ a commutative separable radical Banach algebra which possesses a bounded approximate identity. If the nilpotents are dense in $\mathscr{R}$, there exists for every sequence $\left(\lambda_{n}\right)$ of positive reals an element $c$ of $\mathscr{R}$ such that $[c \mathscr{R}]^{-}=\mathscr{R}$. and $\left\|c^{n}\right\|<\lambda_{n}$ 
for every $n \in N$.

Proof. To prove the theorem we can use any algebra norm over $\mathscr{R}$ which is equivalent to the given one, and it follows from [12] that there exists an equivalent norm over $\mathscr{R}$ for which the bound of the approximate identity is 1 . So we may assume without any loss of generality that there exists a sequence $\left(e_{n}\right)$ of elements of $\mathscr{R}$ such that $\left\|e_{n}\right\|=1$ and

$$
\lim _{n \rightarrow \infty}\left\|\beta e_{n}-\beta\right\|=0
$$

for every $\beta \in \mathscr{R}$. In fact, as $\mathscr{N}$ is dense in $\mathscr{R}$, we may assume that $\left(e_{n}\right)$ is nilpotent for every $n \in N$. Choose any $b \in \mathscr{R}$ such that $[b \mathscr{R}]^{-}=\mathscr{R}$ (the existence of such an element $b$ follows from the Johnson Varopoulos extension of Cohen's factorization theorem [5], [11], [13], as observed by G. R. Allan in [1]). Taking a suitable subsequence of the sequence $\left((1-1 / n) e_{n}\right)$ we easily obtain a sequence $\left(f_{n}\right)$ of nilpotent elements of $\mathscr{R}$ such that $\left\|f_{n}\right\|<1$ and $\left\|b-b f_{n}\right\|<2^{-n}$ for every $n \in N$. Let $\left(q_{n}\right)$ be a strictly increasing sequence of positive integers such that $f_{n}^{q_{n}}=0$ for every $n \in N$. We have, for every

$$
n \in N: \lim _{\substack{n \rightarrow 0 \\ n>0}}\left(\mu e+f_{n}\right)^{k}=0 \text { for every } k \geqq q_{n} .
$$

So we can find for every $n \in N$ a positive real $\mu_{n}$ such that $\left\|\left(\mu_{n} e+f_{n}\right)^{k}\right\|<\lambda_{k}$ if $q_{n} \leqq k<q_{n+1}$ and such that $\left\|\mu_{n} e+f_{n}\right\|<1$, $\left\|b-b\left(\mu_{n} e+f_{n}\right)\right\|<2^{-n}$. Put: $X_{n}=b\left(\mu_{1} e+f_{1}\right) \cdots\left(\mu_{n} e+f_{n}\right)$. We have:

$$
\left\|X_{n}-X_{n+1}\right\| \leqq\left\|\mu_{1} e+f_{1}\right\| \cdots\left\|\mu_{n} e+f_{n}\right\|\left\|b-b\left(\mu_{n+1} e+f_{n+1}\right)\right\|<2^{-n-1} .
$$

So the sequence $\left(X_{n}\right)$ is Cauchy. Denote by $c$ its limit.

Let $k$ be any integer $\geqq q_{1}$. There exists $n \in N$ such that $q_{n} \leqq$ $k<q_{n+1}$. We have, for $m \geqq n$ :

$$
\begin{aligned}
\left\|X_{m}^{k}\right\| & \leqq\left\|\left(\mu_{1} e+f_{1}\right)^{k}\right\| \cdots\left\|\left(\mu_{m} e+f_{m}\right)^{k}\right\|\left\|b^{k}\right\| \\
& \leqq\left\|b^{k}\right\|\left\|\left(\mu_{n} e+f_{n}\right)^{k}\right\| \leqq \lambda_{k}\left\|b^{k}\right\| .
\end{aligned}
$$

So $\left\|c^{k}\right\| \leqq \lambda_{k}$ for $k$ large enough, and taking $\delta>0$ small enough we obtain $\left\|(\delta c)^{k}\right\|<\lambda_{i}$ for every $k \in N$. Also

$$
\begin{aligned}
&\left\|c\left(\mu_{1} e+f_{1}\right)^{-1} \cdots\left(\mu_{n} e+f_{n}\right)^{-1}-b\right\| \\
&= \lim _{m \rightarrow \infty}\left\|b\left(\mu_{n+1} e+f_{n+1}\right) \cdots\left(\mu_{m} e+f_{m}\right)-b\right\| \\
& \leqq\left\|b\left(\mu_{n+1} e+f_{n+1}\right)-b\right\|+\sum_{m=n+1}^{\infty} \| b\left(\mu_{n+1} e+f_{n+1}\right) \cdots \\
& \quad \cdots\left(\mu_{m+1} e+f_{m+1}\right)-b\left(\mu_{n+1} e+f_{n+1}\right) \cdots\left(\mu_{m} e+f_{m}\right) \|
\end{aligned}
$$




$$
\leqq \sum_{m=n+1}^{\infty}\left\|b\left(\mu_{m} e+f_{m}\right)-b\right\| \leqq 2^{-n} .
$$

So $b \in c[\mathscr{R} \oplus C e]^{-}$. As $\mathscr{R}$ has bounded approximate identities, $c \in$ $[c \mathscr{R}]^{-}, \mathscr{R}=[b \mathscr{R}]^{-}=[c \mathscr{R}]^{-}=[(\delta c) \mathscr{R}]^{-}$. This achieves the proof.

Corollary 2.3. Let $A$ be a commutative nonunital complex algebra which is an integral domain of cardinality $2^{\aleph_{0}}$ and let $\mathscr{B}$ be a commutative separable Banach algebra with bounded approximate identities in which the nilpotents are dense. If the continuum hypothesis is assumed there exists for every sequence $\left(\lambda_{n}\right)$ of positive reals a faithful algebra homomorphism $\varphi$ from $A$ into $\mathscr{B}$ such that $\lim _{n \rightarrow \infty} \sup \left\|\varphi\left(a^{n}\right)\right\| / \lambda_{n}=0$ for every nonzero element $a$ of $A$.

Proof. It follows from [9], Corollary 6-2 that if $A$ satisfies the above conditions and if $b \in\left[b^{2} \mathscr{R}\right]^{-}$for some nonnilpotent element of a commutative radical algebra $\mathscr{R}$ then there exists a faithful algebra homomorphism $\varphi$ from $A$ into $\bigcap_{n \in N} b^{n} \cdot \mathscr{R}$ when the continuum hypothesis is assumed. It follows from the Theorem 2.2 that there exists $b \in \mathscr{B}$ such that $[b \mathscr{B}]^{-}=\mathscr{B},\left\|b^{n}\right\|<\lambda_{n}$ for every $n \in N$. Then $b \in\left[b^{2} \mathscr{B}\right]^{-}$and there exists a faithful algebra homomorphism $\varphi$ from $A$ into $\bigcap_{n \in N} b^{n} \mathscr{B}$. As $\lim _{n \in N} \sup \left\|y^{n}\right\| /\left\|b^{n}\right\|=0$ for every $y \in \bigcap_{n \in N} b^{n} \mathscr{B}$, this proves the corollary.

3. Lower estimates of the rates of decrease at infinity for analytic semigroups in commutative radical Banach algebras. Let $\theta$ be any element of $] 0, \pi / 2]$ and let $\mathscr{R}$ be a commutative Banach algebra. We will say that a function $z \rightarrow a^{z}$ over the open angle $U_{\theta}=\{z \in C-\{0\} /|\operatorname{Arg} z|<\theta\}$ is a semigroup in $\mathscr{R}$ defined over $U_{\theta}$ when $a^{z_{1}+z_{2}}=a^{z_{1}} a^{z_{2}}$ for every $z_{1}, z_{2} \in U_{\theta}$, and the semigroup will be said to be analytic when the function $z \rightarrow a^{z}$ is analytic over $U_{\theta}$. Such nonzero semigroups exist an abundance in $\mathscr{R}$ when $\mathscr{R}$ possesses bounded approximate identities. A. M. Sinclair proved in [12] that it is then possible for every $b \in \mathscr{R}$ to construct an analytic semigroup $z \rightarrow a^{z}$ defined over $U_{\pi / 2}$ such that $\left\|a^{z}\right\|$ is bounded over the positive reals and such that $b \in a^{z} \cdot \mathscr{R}$ for every $z \in U_{\pi / 2}$.

We will now give, using the classical Ahlfors Heins theorem, some lower bounds for the rate of decreasing of $\left\|a^{z}\right\|$ along half lines. (The proof works also in the semi-simple case, but our estimates are vacuous if $\mathscr{R}$ is not radical.)

THEOREM 3.1. Let $\mathscr{R}$ be a commutative radical Banach algebra, and let $\theta$ be any element of $] 0, \pi / 2]$. Put: $U_{\theta}=\{z \in C-\{0\} \backslash|\operatorname{Arg} z|<\theta\}$. If a nonzero semigroup $\left(a^{z}\right)_{z \in U \theta}$ in $\mathscr{R}$ is analytic over $U_{\theta}$ we have 


$$
\lim _{r \rightarrow \infty} \log \frac{\left\|a^{\mathrm{re}^{i \varphi}}\right\|}{r^{r}}=0
$$

for every $\gamma>\pi / 2 \theta$ and every $\varphi \in]-\theta, \theta[$, and the convergence is uniform over $[-\alpha, \alpha]$ for every $\alpha \in[0, \theta[$. Similar results hold for $\left\|\psi\left(b a^{z}\right)\right\|$ where $b$ is any element of $\mathscr{R}$ such that $b a \neq 0$ and $\psi$ any homomorphism from $\mathscr{R}$ into a Banach algebra such that $\psi(a b) \neq 0$.

Proof. For $\delta>0$ and $\alpha \in[0, \theta[$ put

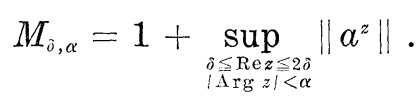

If $|\operatorname{Arg} z| \leqq \alpha, \operatorname{Re} z \geqq 0$ then $|\operatorname{Arg}(\delta+z)| \leqq \alpha$ and there exists $n \in N$ such that $n \delta \leqq \operatorname{Re}(\delta+z)<(n+1) \delta$.

So

$$
\delta \leqq \operatorname{Re}\left(\frac{\delta+z}{n}\right)<\delta+\frac{\delta}{n}, \quad\left|\operatorname{Arg}\left(\frac{\delta+z}{n}\right)\right| \leqq \alpha
$$

and

$$
\left\|a^{(\hat{j}+z) / n}\right\| \leqq M_{\hat{\theta}, \alpha}, \quad\left\|a^{\hat{j}+z}\right\| \leqq\left(M_{\hat{\delta}, \alpha}\right)^{n} \leqq\left(M_{\hat{\delta}, \alpha}\right)^{(|z| / \hat{o}+1} .
$$

Denote by $\log z$ the determination of the logarithm for $z \neq 0$, $\operatorname{Re} z \geqq 0$ which is real over the positive reals and put:

$$
z^{2 \alpha / \pi}=\exp \left[\frac{2 \alpha}{\pi} \log z\right] .
$$

This function can be extended by continuity at zero and is then continuous over $\left[U_{\pi / 2}\right]-$ and analytic over $U_{\pi / 2}$. Let $b$ be any element of $\mathscr{R}$ such that $a b \neq 0$ and let $l$ be a continuous linear from over $\mathscr{R}$ such that $l(a b) \neq 0$. The function

$$
f: z \longrightarrow l\left[b a^{1+z^{2 \alpha / \pi}}\right]
$$

is continuous over $\left[U_{\pi / 2}\right]^{-}$and analytic over $U_{\pi / 2}$. We have, for every $\left.z \in] U_{\pi / 2}\right]^{-}$:

$$
\begin{aligned}
|f(z)| & \leqq\|l\|\|b\|\left(M_{1, \alpha}\right)^{1+|z|^{2 \alpha / \pi}} \\
& \leqq\|l\| \cdot\|b\|\left(M_{1, \alpha}\right)^{2+|z|} .
\end{aligned}
$$

So $f$ is also of exponential type in the closed right-hand half plane $\left[U_{\pi / 2}\right]^{-}$, and $\int_{-\infty}^{\infty} \log ^{+}|f(i y)| /\left(1+y^{2}\right) d y<+\infty$. It follows from the Ahlfors-Heins Theorem (see for example [4], Theorem 7.2.6) that there exists a real number $\lambda$ such that $\lim _{r \rightarrow \infty} \log \left|f\left(\mathrm{re}^{i \varphi}\right)\right| / r=\lambda \cos \phi$ for "many" $\rho$, and certainly for at least one $\rho \in]-\pi / 2, \pi / 2[$. Put: 
$\beta=(2 \alpha \varphi / \pi)$. We obtain

$$
\lim _{r \rightarrow \infty} \frac{\log \left|l\left(b a^{1+\mathrm{re}^{i \beta}}\right)\right|}{\frac{\pi}{2 \alpha}}=\lambda \cos \phi .
$$

So

$$
\begin{aligned}
& \liminf _{r \rightarrow \infty} \frac{\log \left\|b a^{\mathrm{re} i \beta}\right\|}{\frac{\pi}{2 \alpha}} \geqq \lim _{r \rightarrow \infty} \frac{\log \left|l\left(b a^{1+\mathrm{re}^{i} \beta}\right)\right|}{\frac{\pi}{2 \alpha}} \\
& -\lim _{r \rightarrow \infty} \frac{[\log \|l\|+\log \|a\|]}{\frac{\pi}{2 \alpha}}=\lambda \cos \varphi>-\infty .
\end{aligned}
$$

Now let $r$ be any positive real number. We have:

$$
\begin{aligned}
(r+1) e^{i \beta}= & r \cos \beta\left[1-\frac{t g \beta}{t g \alpha}\right] \\
& +\cos \beta\left[1-\frac{t g \beta}{\operatorname{tg} \alpha}\right]+(r+1) \frac{\sin \beta}{\sin \alpha} e^{2 \alpha} .
\end{aligned}
$$

Put:

$$
\delta=\cos \beta\left(1-\frac{\operatorname{tg} \beta}{\operatorname{tg} \alpha}\right), \quad z_{r}=\delta+(r+1) \frac{\sin \beta}{\sin \alpha} e^{i \alpha} .
$$

We have: $\left\|a^{z r}\right\| \leqq M_{\tilde{\delta}, \alpha} \cdot K^{r+1}$, where

$$
K=\left(M_{\delta, \alpha}\right)^{\sin \beta / \delta \sin \alpha} .
$$

So

$$
\left\|b a^{(r+1) e^{i \beta}}\right\| \leqq K^{r+1 \mid} b a^{r \cos \beta(1-t g \beta \mid t g \alpha) \|} \cdot M_{\delta, \alpha}
$$

and

$$
\left\|b a^{(\mu r+1) e^{i \beta}}\right\| \leqq K^{\mu r+1} M_{\hat{o}, \alpha}\left\|b a^{r}\right\|
$$

where

$$
\mu=\frac{\operatorname{tg} \alpha}{\cos \beta(\operatorname{tg} \alpha-\operatorname{tg} \beta)} .
$$

We obtain:

$$
\begin{array}{r}
\liminf _{r \rightarrow \infty} \frac{\log \left\|b a^{r}\right\|}{\frac{\pi}{2 \alpha}} \geqq \liminf _{r \rightarrow \infty} \frac{\log \left\|b a^{(\mu r+1) e^{i \beta}}\right\|}{\frac{\pi}{2 \alpha}} \\
\lim \frac{\log M_{\delta, \alpha}}{\frac{\pi}{2 \alpha}}-\lim _{r \rightarrow \infty} \frac{(r \mu+1) \log K}{\frac{\pi}{2 \alpha}}
\end{array}
$$




$$
=\mu^{2 \pi / \alpha} \liminf _{r \rightarrow \infty} \frac{\log \left\|b a^{(\mu r+1) e^{i \beta}}\right\|}{(\mu r+1)^{\pi / 2 \alpha}}>-\infty .
$$

So

$$
\liminf _{r \rightarrow \infty} \frac{\log \left\|b a^{r}\right\|}{\gamma^{r}}>-\infty \text { for every } \gamma>\frac{\pi}{2 \theta}
$$

which implies in fact that

$$
\liminf _{r \rightarrow \infty} \frac{\log \left\|b a^{r}\right\|}{r^{r}} \geqq 0
$$

for every $\gamma>\pi / 2 \theta$. Now take again $\alpha \in[0, \theta[$ and let $\varphi$ be any element of $[-\alpha, \alpha]$. We have, for every $r>0: 2 \gamma+1=\mathrm{re}^{i \varsigma}+1+$ $z_{r}$, where $z_{r}=r[2-\cos \varphi-i \sin \varphi]$. So

$$
\left|\operatorname{Arg} z_{r}\right| \leqq \Phi \leqq \alpha, \quad\left\|\alpha^{1+z} r\right\| \leqq\left(M_{1, \alpha}\right)^{\mid z z^{\mid+1}} \leqq K_{\alpha}^{r} \cdot M_{1, \alpha}
$$

where

$$
K_{\alpha}=\left(M_{1, \alpha}\right)^{\sqrt{5}}
$$

For $|\operatorname{Arg} z| \leqq \alpha, z \neq 0, \gamma>\pi / 2 \theta$ we obtain:

$$
\begin{aligned}
& \frac{\log \left\|b a^{z}\right\|}{|z|^{\gamma}} \geqq \frac{\log \left\|b a^{2|z|+1}\right\|}{|z|^{\gamma}}-\frac{\log K_{\alpha}}{|z|^{\gamma-1}}-\frac{\log M_{1, \alpha}}{|z|^{\gamma}} \\
& =\left(2+\frac{1}{|z|}\right)^{\gamma} \frac{\log \left\|b a^{2|z|+1}\right\|}{(2|z|+1)^{\gamma}}-\frac{\log K_{\alpha}}{|z|^{\gamma-1}}-\frac{\log M_{1, \alpha}}{|z|^{\gamma}} .
\end{aligned}
$$

Now put:

$$
k_{\alpha}=1+\sup _{\substack{1 \leq|\leq| \leq 2 \\|\Lambda \operatorname{rg} z| \leqq \alpha}}\left\|\alpha^{z}\right\|
$$

If $|z| \geqq 1$, let $n$ be the positive integer satisfying $n \leqq|z|<n+1$. We have:

$$
\left\|b a^{z}\right\| \leqq\|b\|\left\|a^{z / n}\right\|^{n} \leqq\|b\| k_{\alpha}^{n} \leqq k_{\alpha}^{|z|}\|b\| .
$$

Finally we obtain, for $|z| \geqq 1, \gamma>\pi / 2 \theta,|\operatorname{Arg} z| \leqq \alpha$ :

$$
\begin{aligned}
\frac{\log k_{\alpha}}{|z|^{\gamma-1}}+ & \frac{\log \|b\|}{|z|^{\gamma}} \geqq \frac{\log \left\|b a^{z}\right\|}{|z|^{\gamma}} \\
& \geqq\left(2+\frac{1}{|z|}\right)^{\gamma} \frac{\log \left\|b a^{2|z|+1}\right\|}{(2|z|+1)^{\gamma}}-\frac{\log K_{\alpha}}{|z|^{\gamma-1}}-\frac{\log M_{1 . \alpha}}{|z|^{\gamma}} .
\end{aligned}
$$

As

$$
\liminf _{r \rightarrow \infty} \frac{\log \left\|b a^{2 r+1}\right\|}{(2 r+1)^{r}}=0
$$


this proves the first assertion of the theorem. In fact this achieves the proof, because if a semigroup $\left(a^{z}\right)_{z \in U_{\theta}}$ is analytic over $U_{\theta}$ then the $\operatorname{map} z \rightarrow \psi\left(a^{z}\right)$ defines an analytic semigroup over $U_{\theta}$ in $\mathscr{B}$ if $\psi$ is any continuous homomorphism from $\mathscr{R}$ into a Banach algebra $\mathscr{B}$.

COROLlaRy 3.2. Let $a$ be a nonzero element of a commutative radical Banach algebra $\mathscr{R}$. If there exists an analytic semigroup $z \rightarrow a^{z}$ defined over $U_{\theta}$ for some $\theta \in[0, \pi / 2]$ then

$$
\liminf _{r \rightarrow \infty}\left[\exp \left(n^{\gamma-1}\right)\right]\left\|a^{n}\right\|^{1 / n}=+\infty \quad \text { for every } \quad \gamma>\frac{\pi}{2 \theta} .
$$

Proof. Let $\gamma$ be any element of $] \pi / 2 \theta\left[, \infty\left[\right.\right.$ and let $\gamma^{\prime}$ be an element of $] \pi / 2 \theta, \gamma[$. There exists $m>0$ such that

$$
\left\|a^{n}\right\|^{1 / n} \geqq \exp \left[-m n^{\gamma^{\prime}-1}\right] \text {, }
$$

for every $n \in N$. So

$$
\begin{aligned}
& \lim \underset{n \rightarrow \infty}{\exp }\left[n^{\gamma-1}\right]\left\|a_{n}\right\|^{1 / n} \\
& \quad \geqq \liminf _{n \rightarrow \infty} \exp \left[n^{\gamma-1}-m n^{r^{\prime-1}}\right]=+\infty .
\end{aligned}
$$

We now give lower estimates for the rate of decrease at infinity of $\left\|a^{r}\right\|$, where $r \rightarrow a^{r}$ is an analytic semigroup over $] 0,+\infty[$.

THEOREM 3.3. Let $\mathscr{R}$ be a commutative radical Banach algebra and let $\left(a^{r}\right)_{r>0}$ be a semigroup in $\mathscr{R}$ defined over the positive reals. If the semigroup is analytic over $] 0,+\infty[$ then there exists $\lambda>0$ such that

$$
\lim _{r \rightarrow \infty} \log \left\|a^{r}\right\| \exp (-\lambda r)=0
$$

(and more generally such that

$$
\lim _{r \rightarrow \infty} \log \left\|\psi\left(b a^{r}\right)\right\| \exp (-\lambda r)=0
$$

for every $b \in \mathscr{R}$ and for every homomorphism is from $\mathscr{R}$ into a Banach algebra satisfying $\psi(a b) \neq 0$ ).

Proof. The function $r \rightarrow a^{r}$ is analytic at 1 , so there exists $\eta>0$ and a sequence $\left(a_{n}\right)_{n \geqq 0}$ of elements of $\mathscr{R}$ such that

$$
a^{r}=\sum_{n=0}^{\infty} a_{n}(r-1)^{n} \quad \text { for every } \quad r \in[1-2 \eta, 1+2 \eta] .
$$

So the series 


$$
\sum_{n=0}^{\infty} a_{n} z^{n}
$$

is absolutely convergent for $|z| \leqq 2 \eta$, and its sum $f(z)$ is analytic for $|z|<2 \eta$.

We have, for every $t_{1}, t_{2} \in[-\eta, \eta] f\left(t_{1}\right) f\left(t_{2}\right)=a^{1+t_{1}} a^{1+t_{2}}=a f\left(t_{1}+t_{2}\right)$. This implies that the equality $f\left(z_{1}\right) f\left(z_{2}\right)=a f\left(z_{1}+z_{2}\right)$ holds for every $z_{1}, z_{2} \in C$ such that $\left|z_{1}\right| \leqq \eta,\left|z_{2}\right| \leqq \eta$. Now take any $z \in C$ such that $\operatorname{Re} z \geqq 2,|\operatorname{Im} z| \leqq \eta$. Put: $g(z)=f[i \operatorname{Im} z] a^{\operatorname{Re} z-1}$. Using the analyticity of $f$ and the multiplicative properties of the semigroup we see easily that $g$ is analytic in the interior of the set $\Delta=\{z \in$ $C / \operatorname{Re} z \geqq 2, \operatorname{Im} z \leqq \eta\}$ and continuous over $\Delta$. If $z_{1}=x_{1}+i y_{1} \in \Delta$, $z_{2}=x_{2}+i y_{2} \in \Delta$ and if the sum $z_{1}+z_{2}$ belongs also to $\Delta$ we have:

$$
\begin{aligned}
g\left(z_{1}+z_{2}\right) & =f\left(i y_{1}+i y_{2}\right) a^{x_{1}+x_{2}-1} \\
& =a f\left(i y_{1}+i y_{2}\right) a^{x_{1}+x_{2}-2} \\
& =f\left(i y_{1}\right) f\left(i y_{2}\right) a^{x_{1}+x_{2}-2} \\
& =g\left(z_{1}\right) g\left(z_{2}\right) .
\end{aligned}
$$

Also if $z$ is real and if $\operatorname{Re} z \geqq 2$ we have $g(z)=f(0) a^{z-1}=a \cdot a^{z-1}=a^{z}$.

For $\operatorname{Re} z>0$ denote by $\log z$ the determination of the logarithm which takes real values over the positive reals. The function

$$
z \longrightarrow 2+\frac{2 \eta}{\pi} \log (z+1)
$$

maps the closed right-hand half plane $\left[U_{\pi / 2}\right]^{-}$into $\Delta$. Now let $b$ be an element of $\mathscr{R}$ such that $a b \neq 0$, let $\psi$ be a continuous homomorphism from $\mathscr{B}$ into a Banach algebra $\mathscr{B}$ and let $l$ be a continuous linear form over $\mathscr{B}$ such that $l[\psi(a b)] \neq 0$. The function

$$
h: z \longrightarrow l\left[\psi\left[b \cdot g\left(2+\frac{2 \eta}{\pi} \log (z+1)\right)\right]\right]
$$

is analytic over $U_{\pi / 2}$ and continuous over $\left[U_{\pi / 2}\right]^{-}$. As

$$
\lim _{n \rightarrow \infty}\left\|a^{n}\right\|=0 \text {, }
$$

there exists $M>0$ such that $\left\|a^{x}\right\| \leqq M$ for every $x \in[1,+\infty$ [, which implies that $g$ is bounded over $\Delta$. So $h$ is bounded over $\left[U_{\pi / 2}\right]^{-}$.

Using again the Ahlfors-Heins theorem we see that

$$
\liminf _{r \rightarrow \infty} \frac{\log \left|h\left(\mathrm{re}^{i \theta}\right)\right|}{r}>-\infty \text { for some } \theta \in\left[0, \frac{\pi}{2}[.\right.
$$




$$
\begin{aligned}
& \liminf _{r \rightarrow \infty} \frac{\log \left\|\psi\left[b \cdot g\left[2+\frac{2 \eta}{\pi} \log \left(1+\mathrm{re}^{i \theta}\right)\right]\right]\right\|}{r}>-\infty \text { for some } \\
& \theta \in\left[0, \frac{\pi}{2}[.\right.
\end{aligned}
$$

We have:

$$
g\left[2+\frac{2 \eta}{\pi} \log \left(1+\mathrm{re}^{i \theta}\right)\right]=f\left[\frac{2 i \eta}{\pi} \operatorname{Arg}\left(1+\mathrm{re}^{i \theta}\right)\right] a^{1+2 \eta / \pi \log \mid 1+\mathrm{r} \mathrm{r}^{i \theta \mid}} .
$$

Put:

$$
K=\sup _{|z|<\eta}|f(z)|
$$

We obtain:

$$
\left\|\psi\left(b g\left(2+\frac{2 \eta}{\pi}\left(\log \left(1+\mathrm{re}^{i \theta}\right)\right)\right)\right)\right\| \leqq K\|\psi\|\left\|\psi\left(b a^{1+2 \eta / \pi \log \mid 1+\mathrm{r} \mathrm{e}^{i \theta \mid}}\right)\right\| .
$$

For $r>1, \log \left|1+\mathrm{re}^{i \theta}\right| \geqq \log (r-1)$ and

$$
1+\frac{2 \eta}{\pi} \log \left|1+\mathrm{re}^{i \theta}\right|=\frac{2 \eta}{\pi} \log (r-1)+x_{r},
$$

where $x_{r} \geqq 1$. We obtain, for every $r>1$ :

$$
\left\|\psi\left[b g\left(2+\frac{2 \eta}{\pi} \log \left(1+\mathrm{re}^{i \theta}\right)\right)\right]\right\| \leqq K M\|\psi\|^{2}\left\|\psi\left[b a^{2 \eta / \pi \log (r-1)}\right]\right\| .
$$

So

$$
\liminf _{r \rightarrow \infty} \frac{\log \left\|\psi\left[b a^{2 \eta / \pi \log (r-1)}\right]\right\|}{r}>-\infty
$$

$$
\begin{gathered}
\liminf _{t \rightarrow \infty}\left[\log \left\|\psi\left[b a^{t}\right]\right\|\right] \exp \left[-\frac{\pi}{2 \eta} t\right]=\liminf _{t \rightarrow \infty} \frac{\log \left\|\psi\left(b a^{t}\right)\right\|}{1+\exp \left[\frac{\pi}{2 \eta} t\right]} \\
=\liminf _{r \rightarrow \infty} \frac{\log \left\|\psi\left(b a^{2 \eta / \pi \log (r-1)}\right)\right\|}{r}>-\infty
\end{gathered}
$$

So

$$
\liminf _{t \rightarrow \infty}\left[\log \left\|\psi\left(b a^{t}\right)\right\|\right] \exp \left[-\frac{\pi}{\eta} t\right] \geqq 0
$$

As

$$
\lim _{t \rightarrow \infty}\left\|\psi\left(b a^{t}\right)\right\|=0, \quad \limsup _{t \rightarrow \infty}\left[\log \left\|\psi\left(b a^{t}\right)\right\|\right] \exp \left[-\frac{\pi}{\eta} t\right] \leqq 0
$$


So

$$
\lim _{t \rightarrow \infty}\left[\log \left\|\psi\left(b a^{t}\right)\right\|\right] \exp \left[-\frac{\pi}{\eta} t\right]=0
$$

which proves the theorem.

COROLlary 3.4. Let $\mathscr{R}$ be a commutative radical Banach algebra, and let $a$ be a nonzero element of $\mathscr{R}$. If there exists an analytic semigroup $\left(a^{r}\right)_{r>0}$ over $] 0,+\infty\left[\right.$ with $a^{1}=a$, then

$$
\lim \inf \left\|a^{n}\right\| \exp [\exp (\lambda n)]=+\infty \text { for some } \lambda>0 .
$$

Proof. It follows from the theorem that there exists two positive numbers $c$ and $\mu$ such that $\log \left\|a^{n}\right\| \geqq-c \exp [\mu n]$ for every $n \geqq 1$. So $\left\|a^{n}\right\| \geqq \exp [-c \exp (\mu n)]$ for every $n \geqq 1$ and

$\liminf _{n \rightarrow \infty}\left\|a^{n}\right\| \exp [\exp (2 \mu n)] \geqq \liminf _{n \rightarrow \infty} \exp \left[\exp (2 \mu n)-c \exp \left(\mu_{n}\right)\right]=+\infty$.

REMARK 3.5. In the proofs of the Theorems 3.1 and 3.3 we only used the fact that the map $z \mapsto l\left(b a^{z}\right)$ is analytic for at least one continuous linear form $l$ over $\mathscr{R}$ such that $l(a b) \neq 0$, so the theorems remain true under this weaker notion of analyticity. Note also that in the proof of the Theorem 3.3 we only used the fact that the function $r \rightarrow a^{r}$ is analytic at 1 . In particular if $\lim _{n \rightarrow \infty}\left\|b^{n}\right\| \exp [\exp$ $(n \log n)]=0$ for every nonzero element $b$ of a commutative radical Banach algebra $\mathscr{R}$ then the only semigroup $\left(a^{r}\right)_{r>0}$ which is analytic at 1 is the zero semigroup.

We will now show that there are no general lower bounds for the rate of decrease at infinity of the function $t \rightarrow\left\|a^{t}\right\|$ for infinitely differentiable semigroups $\left(a^{t}\right)_{t>0}$ over $] 0,+\infty\left[\right.$ such that $a^{t} \neq 0$ for every $t>0$.

Lemma 3.4. For $\alpha>0$, denote by $f_{\alpha}$ the function defined over $[0,+\infty[$ by the formula:

$$
\begin{gathered}
f_{\alpha}(x)=0 \text { if } x \leqq \alpha \\
f_{\alpha}(x)=\frac{\alpha}{\sqrt{\pi}(x-\alpha)^{3 / 2}} \exp \left(-\frac{\alpha^{2}}{x-\alpha}\right) \text { if } x>\alpha .
\end{gathered}
$$

The function $\alpha \rightarrow f_{\alpha}$ defines in $L^{1}\left(R^{+}\right)$an infinitely differentiable semigroup over the positive reals.

Proof. Put: 


$$
g_{\alpha}(x)=\frac{\alpha}{\sqrt{\pi} x^{3 / 2}} \exp \left(-\frac{\alpha^{2}}{x}\right)
$$

for $x>0$ and $g_{\alpha}(0)=0$. Then $f_{\alpha}=g_{\alpha} * \delta_{\alpha}$, where $\delta_{\alpha}$ is the point measure at $\alpha$. A calculation given in [7], p. 63 shows that the Laplace transform of $g_{1}$ is the function $z \mapsto \exp (-2 \sqrt{z})$ where $\sqrt{z}$ is the detemination of the square root over the closed right half plane which takes real positive values over the positive reals.

We have:

$$
\mathscr{L}\left[g_{\alpha}\right](z)=\int_{0}^{\infty} g_{\alpha}(x) \exp (-z x) d x
$$

Put: $u=x / \alpha^{2}$. We obtain:

$\mathscr{L}\left[g_{\alpha}\right](z)=\int_{0}^{\infty} \frac{\exp \left(-u^{2}\right) \exp \left(-\alpha^{2} z u\right)}{\sqrt{\pi} u^{3 / 2}} d u=\mathscr{L}\left(g_{1}\right]\left[\alpha^{2} z\right]=\exp [-2 \alpha \sqrt{z}]$

So $\mathscr{L}\left[g_{\alpha+\beta}\right]=\mathscr{L}\left(g_{\alpha}\right) \mathscr{L}\left(g_{\beta}\right)=\mathscr{L}\left(g_{\alpha} * g_{\beta}\right)$ and using the uniqueness theorem for Laplace transforms we see that $g_{\alpha+\beta}=g_{\alpha} * g_{\beta}$ for every $\alpha, \beta \in] 0,+\infty\left[\right.$. So $f_{\alpha+\beta}=g_{\alpha+\beta} * \delta_{\alpha+\beta}=\left(g_{\alpha} * \delta_{\alpha}\right) *\left(g_{\beta} * \delta_{\beta}\right)=f_{\alpha} * f_{\beta}$ for every $\alpha, \beta \in] 0,+\infty\left[\right.$. So the map $\alpha \mapsto f_{\alpha}$ defines in $L^{1}\left(R^{+}\right)$a semigroup over the positive reals.

It is easy to see that the $n$ th-derivative of $f_{\alpha}$ with respect to $\alpha$ has the form $f_{\alpha}^{(n)}[x]=0$ if $x \leqq \alpha, f_{\alpha}^{(n)}[x]=v_{n}(\alpha)[x] \exp \left[-\alpha^{2} / x\right]$ if $x>\alpha$ where

$$
v_{n}(\alpha)(x)=\frac{P_{n}(\alpha, x)}{(x-\alpha)^{3 / 2+2 n}}
$$

for some polynomial $P_{n}(\alpha, x)$ in two variables whose degree with respect to $x$ is $2 n$. So $f_{\alpha}^{(n)} \in L^{1}\left(R^{+}\right)$for every $\alpha>0$ and every $n \in N$ and it is not difficult to prove that $f_{\alpha}^{(n)}(x)$ depends continuously of $\alpha$ for every $x \geqq 0$ and every $n \in N$. Also for every $\alpha>0, \eta \in] 0, \alpha[$ and every $n \in N$ there exists a continuous positive integrable function $u_{n, \alpha, \eta}$ over $\left[0,+\infty\left[\operatorname{such}\right.\right.$ that $\left|f_{\beta}^{(n)}(x)\right| \leqq u_{n, \alpha, \eta}$ for every $x \geqq 0$ and every $\beta \in] \alpha-\eta, \alpha+\eta[$. We have

$$
\begin{aligned}
& \left\|\frac{f^{(n)}(\alpha+h)-f^{(n)}(\alpha)}{h}-f^{(n+1)}(\alpha)\right\| \\
& \quad \leqq \int_{0}^{\infty}\left|f_{\alpha+\theta_{\alpha}^{(h, x) h}}^{(n+1)}(x)-f_{\alpha}^{(n+1)}(x)\right| d x
\end{aligned}
$$

where $0<\theta_{\alpha}(h, x)<1$. Let $\left(h_{p}\right)$ be any sequence of nonzero real numbers such that $\lim _{p \rightarrow \infty} h_{p}=0$. We have, for $p$ large enough:

$$
\left|f_{\alpha+\theta}^{(n+1)}\left(h_{p}, x\right) h_{p}(x)-f_{\alpha}^{(n+1)}(x)\right| \leqq 2 u_{n, \alpha, \alpha / 2}(x)
$$


for every $x \geqq 0$. As $\lim _{p \rightarrow \infty} \theta_{\alpha}\left(h_{p}, x\right) h_{p}=0$ for every $x \geqq 0$, it follows from the dominated convergence theorem that

$$
\lim _{p \rightarrow \infty}\left\|\frac{f_{\alpha+h_{p}}^{(n)}-f_{\alpha}^{(n)}}{h_{p}}-f_{\alpha}^{(n+1)}\right\|=0 .
$$

So

$$
\lim _{h \rightarrow \infty}\left\|\frac{f_{\alpha+h}^{(n)}-f_{\alpha}^{(n)}}{h}-f_{\alpha}^{(n+1)}\right\|=0
$$

and the semigroup $\left(f_{\alpha}\right)_{\alpha>0}$ is infinitely differentiable, which proves the lemma.

THEOREM 3.6. Let $g$ be any continuous positive function over $[1, \infty[$ such that $g(1)=1$. There exists a positive continuous function $\omega$ over $[0,+\infty[$ such that $\omega(x+y) \leqq \omega(x) \omega(y)$ for every $x, y \geqq 0$, $\lim _{x \rightarrow \infty}[\omega(x)]^{1 / x}=0$ and a semigroup $\left(a^{t}\right)_{t>0}$ in $L^{1}\left(R^{+}, \omega\right)$ which is infinitely differentiable over $\left[0, \infty\left[\right.\right.$ and satisfies $0<\left\|a^{t}\right\|<g(t)$ for every $t \geqq 1$.

Proof. Let $l$ be the positive continuous function over $[0,+\infty[$ defined by the formula:

$$
\begin{array}{lll}
l(x)=1 & \text { if } & 0 \leqq x \leqq 1 \\
l(x)=\inf (1, g(x)) & \text { if } & x \geqq 1 .
\end{array}
$$

Put, for $x \geqq 0: \varphi(x)=\inf l\left(y_{1}\right) \cdots l\left(y_{n}\right)$ the infimum being taken over all finite families $\left(y_{1}, \cdots, y_{n}\right)$ of nonnegative real numbers such that $x=y_{1}+\cdots+y_{n}$. It follows immediately from the definition of $\varphi$ that $0 \leqq \varphi(x) \leqq 1$ for every $x \geqq 0, \varphi(x) \leqq g(x)$ for every $x \geqq 1$ and $\varphi(x+y) \leqq \varphi(x) \varphi(y)$ for every $x, y \geqq 0$. Let $x \geqq 0$ and let $\left(y_{1}, \cdots, y_{n}\right)$ be any family of nonnegative reals such that $x=y_{1}+\cdots+y_{n}$. Denote by $p_{x}$ the greatest integer such that $p_{x} \leqq x$. At most $p_{x}$ elements of the family $\left(y_{1}, \cdots, y_{n}\right)$ are greater than 1 , so

$$
l\left(y_{1}\right) \cdots l\left(y_{n}\right) \geqq[m(x)]^{p_{x}}
$$

where

$$
m(x)=\inf _{0 \leqq y \leq x} l(y)
$$

So $\varphi(x)>0$.

Also for every $\delta>0$ we have:

$$
\varphi(\delta+x) \leqq l(\delta) l\left(y_{1}\right) \cdots l\left(y_{n}\right) \leqq l\left(y_{1}\right) \cdots l\left(y_{n}\right) .
$$

As this inequality holds for every family $\left(y_{1}, \cdots, y_{n}\right)$ of nonnegative reals such that $x=y_{1}+\cdots+y_{n}$, we have $\varphi(\delta+x) \leqq \varphi(x)$ and $\varphi$ is 
decreasing over $[0,+\infty[$. Now let $m \in N$ and let $\varepsilon>0$. There exists $\alpha>0$ such that $\left|l\left(y_{1}\right)-l\left(y_{2}\right)\right|<\varepsilon / 2$ for every $y_{1}, y_{2} \in[0, m]$ satisfying $\left|y_{1}-y_{2}\right|<\alpha$, and we may assume $\alpha<1$. Let $x_{1}, x_{2}$ be two elements of $[0, m]$ such that $0 \leqq x_{2}-x_{1}<\alpha$. If $\varphi\left(x_{2}\right)=1$ then $\varphi\left(x_{1}\right)=1, \varphi\left(x_{2}\right)-\varphi\left(x_{1}\right)=0$. If $\varphi\left(x_{2}\right)<1$ there exists a finite family $\left(y_{1}, \cdots, y_{n}\right)$ of nonnegative reals such that

$$
x_{2}=y_{1}+\cdots+y_{n}, l\left(y_{1}\right) \cdots l\left(y_{n}\right)<\inf \left[\frac{1+\varphi\left(x_{2}\right)}{2}, \varphi\left(x_{2}\right)+\frac{\varepsilon}{2}\right] .
$$

So $y_{2}>1$ for at least one $y_{i}$, say $y_{n}$. Then $y_{n}-\left(x_{2}-x_{1}\right)>0$ and we have:

$$
\begin{aligned}
& x_{1}=y_{1} \cdots+y_{n-1}+\left[y_{n}-\left(x_{2}-x_{1}\right)\right], \\
& \varphi\left(x_{1}\right) \leqq l\left(y_{1}\right) \cdots l\left(y_{n-1}\right)\left[l\left(y_{n}\right)+\frac{\varepsilon}{2}\right] \\
& \leqq l\left(y_{1}\right) \cdots l\left(y_{n}\right)+\frac{\varepsilon}{2}<\varphi\left(x_{2}\right)+\varepsilon .
\end{aligned}
$$

So $0 \leqq \varphi\left(x_{2}\right)-\varphi\left(x_{1}\right)<\varepsilon$ and $\varphi$ is uniformly continuous over $[0, m]$ for every $m \in N$, which shows that $\varphi$ is continuous over [0, $+\infty$ [. Put, for $x \geqq 0: \omega(x)=\varphi(x) \exp \left(-x^{2}\right)$. Then $\omega(x+y) \leqq \omega(x) \omega(y)$ for every $x, y \in[0,+\infty]$, and

$$
\limsup _{x \rightarrow \infty}[\omega(x)]^{1 / x} \leqq \limsup _{x \rightarrow \infty} e^{-x}=0,
$$

$0<\omega(x) \leqq 1$ for every $x \geqq 0, \omega(x) \leqq g(x)$ for every $x \geqq 1$ and $\omega$ is continuous over $[0,+\infty[$.

Clearly $L^{1}\left(R^{+}\right) \subseteq L^{1}\left(R^{+}, \omega\right)$. Put, for $t>0: a^{t}=f_{t}$, where $f_{t}$ is the function defined in the lemma. As the injection from $L^{1}\left(R^{+}\right)$ into $L^{1}\left(R^{+}, \omega\right)$ is continuous, the semigroup $\left(a^{t}\right)_{t>0}$ is infinitely differentiable over $\left[0,+\infty\left[\right.\right.$ with respect to the norm of $L^{1}\left(R^{+}, \omega\right)$.

Also

$$
\begin{aligned}
\left\|a^{t}\right\| & =\int_{t}^{\infty} \frac{t}{\sqrt{\pi}(x-t)^{3 / 2}} \exp \left(\frac{-t^{2}}{x-t}\right) \omega(x) d x \\
& \leqq \omega(t) \int_{t}^{\infty} \frac{t}{\sqrt{\pi}(x-t)^{2 / 2}} \exp \left(\frac{-t^{2}}{x-t}\right) d x \\
& =\omega(t) \int_{0}^{\infty} \frac{t}{\sqrt{\pi} x^{3 / 2}} \exp \left(\frac{-t^{2}}{x}\right) d x=\omega(t) \mathscr{L}\left(g_{t}\right)(0),
\end{aligned}
$$

where $g_{t}$ is the function

$$
x \longrightarrow \frac{t}{\sqrt{\pi} x^{3 / 2}} \exp \frac{-t^{2}}{x} .
$$


As $\mathscr{L}\left(g_{t}\right)(z)=\exp (-2 t \sqrt{z})$, we obtain: $\left\|a^{t}\right\| \leqq \omega(t)$ for every $t>0$. So $\left\|a^{t}\right\| \leqq g(t)$ for every $t \geqq 1$, which proves the theorem.

REMARK 3.7. The example of the Theorem 3.6 has the property that $a^{t} \notin\left[a^{t^{\prime}} L^{1}\left(R^{+}, \omega\right)\right]^{-}$if $t>t^{\prime}$. It is also possible, given a positive continuous function $g$ over $[1,+\infty$ [, to find a continuous weight $\omega$ and an infinitely differentiable semigroup $\left(a^{t}\right)_{t>0}$ over ]0, $+\infty[$ in $L^{1}\left(R^{+}, \omega\right)$ such that $0<\left\|a^{t}\right\|<g(t)$ for every $t \geqq 1$ and such that $\left[a^{t} L^{1}\left(R^{+}, \omega\right)\right]^{-}=L^{1}\left(R^{+}, \omega\right)$ for every $t>0$, but these examples involve much more difficult computations (which will be given in [10]) and we will not do this here.

4. Lower bounds for the rate of decrease of sequences of powers in weighted convolution algebras.

THEOREM 4.1. Let $\mathscr{R}$ be a commutative radical Banach algebra without nilpotent elements. If the map $a \mapsto b a$ is a compact endomorphism of $\mathscr{R}$ for some element $b$ of $\mathscr{R}$ which is not a divisor of 0 then for every continuous algebra norm $q$ over $\mathscr{R}$ there exists a sequence $\left(\alpha_{n}\right)_{n \geqq 0}$ of positive real numbers (the sequence depending on the norm) such that

$$
\liminf _{n \rightarrow \infty} \frac{q\left(\alpha^{n}\right)}{\alpha_{n}}=+\infty
$$

for every nonzero element a of $\mathscr{R}$.

Proof. For $p \in N$, put: $E_{p}=\{a \in \mathscr{R} \mid\|b a\|>1 / p\|a\|\}$. Put, for $n \in N$ :

$$
\lambda_{n, p}=\inf _{a \in E_{p}} \frac{q\left(a^{n}\right)}{\|a\|^{n}} .
$$

Suppose that $\lambda_{n, p}=0$ for some $n, p \in N$.

Then there exists a sequence $\left(a_{k e}\right)_{k \in N}$ of elements of $E_{p}$ such that $\left\|a_{k}\right\|=1$ for every $k \in N$ and $\lim _{k \rightarrow \infty} q\left(a_{k}^{n}\right)=0$.

As $b$ acts compactly in $\mathscr{R}$, it would be possible to find a subsequence

$$
\left(a_{k_{i}}\right)_{i \in N}
$$

of $\alpha_{k}$ such that the sequence

$$
\left(b a_{k_{i}}\right)_{\imath \in N}
$$

is convergent. Denote by $c$ its limit. As $a_{k} \in E_{p}$ for every $k \in N$, 


$$
\|b c\|=\lim _{n \rightarrow \infty}\left\|b a_{k_{i}}\right\| \geqq \frac{1}{p}, \quad \text { so } \quad c \neq 0 \text {. }
$$

Also

$$
q\left(c^{n}\right)=\lim _{i \rightarrow \infty} q\left(b^{n} a_{k_{i}}^{n}\right) \leqq q\left(b^{n}\right) \lim _{i \rightarrow \infty} q\left(a_{k_{i}}^{n}\right)=0, \quad c^{n}=0
$$

and $c$ would be nilpotent, which is impossible.

So $\lambda_{n, p}>0$ for every $n, p \in N$, and the family

$$
\left(\left(n^{-n} \lambda_{n, p}\right)_{n \in N}\right)_{p \in N}
$$

is a countable family of sequences of positive reals. It is then wellknown that there exists another sequence $\left(\alpha_{n}\right)_{n \in N}$ of positive reals satisfying

$$
\liminf _{n \rightarrow \infty} \frac{n^{-n} \lambda_{n, p}}{\alpha_{n}}=+\infty
$$

for every $p \in N$. Let $a$ be any nonzero element of $\mathscr{R}$. As $b$ is not a divisor of zero, $b a \neq 0$ and $a \in E_{p}$ for some $p \in N$. We obtain:

$$
\begin{aligned}
\liminf _{n \rightarrow \infty} \frac{q\left(a^{n}\right)}{\alpha_{n}} & \geqq \liminf _{n \rightarrow \infty} \frac{q\left(a^{n}\right)}{\|a\|^{n} \cdot \lambda_{n, p}} \cdot[n\|a\|]^{n} \cdot \frac{\left(n^{-n} \cdot \lambda_{n, p}\right)}{\alpha_{n}} \\
& \geqq \liminf _{n \rightarrow \infty}(n\|a\|)^{n} \cdot\left(\frac{n^{-n} \cdot \lambda_{n, p}}{\alpha_{n}}\right) \\
& \geqq \liminf _{n \rightarrow \infty}\left(\frac{n^{-n} \cdot \lambda_{n, p}}{\alpha_{n}}\right)=+\infty .
\end{aligned}
$$

This proves the theorem.

CoRollaRY 4.2. Let $\omega$ be a positive continuous function over ] $0,+\infty[$ such that $\omega(x+y) \leqq \omega(x) \omega(y)$ for every $x, y \in] 0,+\infty[$, $\lim _{x \rightarrow \infty}[\omega(x)]^{1 / x}=0$. There exists a sequence $\left(\alpha_{n}(\omega)\right)_{n \in N}$ of positive reals such that

$$
\liminf _{n \rightarrow \infty} \frac{\left\|a^{n}\right\|}{\alpha_{n}(\omega)}=+\infty
$$

for every nonzero element a of $L^{1}\left(R^{+}, \omega\right)$.

Proof. W. G. Badé and H. G. Dales discussed in [3] the compactness of the map $f \rightarrow f * g$ in $L^{1}\left(R^{+}, \omega\right)$ where $\omega$ is a continuous weight over $[0,+\infty$ [ satisfying $\omega(x+y) \leqq \omega(x) \omega(y)$ for all $x, y \in$ $[0,+\infty[$ and

$$
\lim _{x \rightarrow \infty}[\omega(x)]^{1 / x}=0
$$


Their result is that this map is compact iff

$$
\lim _{x \rightarrow \infty} \int_{0}^{\infty}|g(y)| \frac{\omega(x+y)}{\omega(x)} d y=0 .
$$

In particular if $\omega$ is a continuous weight over $[0,+\infty$ [ satisfying the above conditions then every element in $L^{1}\left(R^{+}, \omega\right)$ acts compactly provided the function $x \mapsto[\omega(x)]^{1 / x}$ is descreasing over $[0,+\infty[$.

Now let $\omega$ be any continuous weight over $] 0,+\infty[$, not necessarily bounded at the origin. Using the same argument as in the proof of the Theorem 3.6 we can construct a decreasing positive continuous function $h$ over $[0,+\infty$ [ such that $h(x) \leqq 1$ for every $x \geqq 0, h(x+y) \leqq h(x) h(y)$ for every $x, y \geqq 0$ and $h(x) \leqq \omega(x)$ for every $x \geqq 1$. Put: $v(x)=[h(x)]^{x} ; v$ is positive and continuous over $[0,+\infty[$,

$$
\begin{gathered}
\lim _{x \rightarrow \infty}[v(x)]^{1 / x}=\lim _{x \rightarrow \infty} h(x)=0, \\
v(x+y)=[h(x+y)]^{x+y} \leqq[h(x)]^{x} \cdot[h(y)]^{y}=v(x) v(y)
\end{gathered}
$$

for every $x, y>0$. So $L^{1}\left(R^{+}, v\right)$ is a commutative radical Banach algebra and Bade's theorem implies that all elements of $L^{1}\left(R^{+}, v\right)$ act compactly in $L^{1}\left(R^{+}, v\right)$. Also $L^{1}\left(R^{+}, v\right)$ is an integral domain (this is an easy consequence of Titchmarsh's convolution theorem, see [6]). So there exists a sequence $\left(\alpha_{n}\right)$ of positive reals such that

$$
\liminf _{n \rightarrow \infty} \frac{\left\|f^{n}\right\|_{v}}{\alpha_{n}}=+\infty
$$

for every nonzero $f \in L^{1}\left(R^{+}, v\right)$ (where we denote by $\|\cdot\|_{v}$ the norm in $L^{1}\left(R^{+}, v\right)$ ). As $\omega$ is bounded below at the origin, the quotient $(v(x) / \omega(x))$ is bounded over $[0,1]$ and $v(x)=[h(x)]^{x} \leqq h(x) \leqq \omega(x)$ for every $x \geqq 1$. So $L^{1}\left(R^{+}, \omega\right) \subseteq L^{1}\left(R^{+}, v\right)$ and this injection is continuous, which shows that the sequence $\left(\alpha_{n}\right)$ defined above satisfies the condition of the theorem.

Note that the Corollary 4.2 is in fact true for every continuous

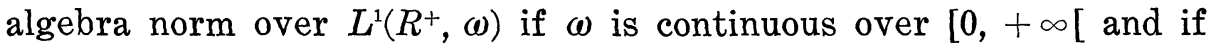
the function $x \mapsto[\omega(x)]^{1 / x}$ is decreasing over $[0,+\infty[$. Also the corollary can be extended to every measurable weight over $[0,+\infty[$ which does not vanish almost everywhere over $[a,+\infty[$ for any $a>0$.

REMARK 4.3. The Theorem 4.1 is true for commutative separable radical Banach algebras without nilpotent elements in which every element acts compactly: let $\left(a_{m}\right)$ be a dense sequence in such a Banach algebra $\mathscr{R}$. The argument used in the proof of the 
Theorem 4.1 shows that for every $m \in N$ and every continuous algebra norm $q$ over $\mathscr{R}$ there exists a sequence $\left(\alpha_{n, m}\right)_{n \in N}$ of positive reals such that

$$
\liminf _{n \rightarrow \infty} \frac{q\left(b^{n}\right)}{\alpha_{n, m}}=+\infty
$$

for every $b \in \mathscr{R}$ such that $b \cdot a_{m} \neq 0$. There exists a sequence $\left(\lambda_{n}\right)_{n \in N}$ of positive real numbers such that

$$
\liminf _{n \rightarrow \infty} \frac{\alpha_{n, m}}{\lambda_{n}}=+\infty
$$

for every $m \in N$. Now let $b$ be any nonzero element of $\mathscr{R}$. Then $b \cdot a_{m} \neq 0$ for some $m \in N$, for otherwise $b \cdot \mathscr{R}=0, b^{2}=0$ and $b$ would be nilpotent. So

$$
\liminf _{n \rightarrow \infty} \frac{q\left(b^{n}\right)}{\lambda_{n}}=0,
$$

which proves our assertion.

This result does not extend to nonseparable commutative Banach algebras in which every element acts compactly (take the direct sum $\mathscr{R}$ of all algebras $L^{1}\left(R^{+}, \omega\right)$ where $\omega$ runs over the set of all positive continuous weights over $[0, \infty[$ such that $\omega(x+y) \leqq \omega(x) \omega(y)$ for all $x, y \geqq 0$ and $\lim _{x \rightarrow \infty}[\omega(x)]^{1 / x}=0$ ).

\section{REFERENCES}

1. G. R. Allan, Embedding the algebra of all formal power series in a Banach algebra, Proc. London Math. Soc., (3) 25 (1972), 329-340.

2. G. R. Allan and A.M. Sinclair, Power factorization in Banach algebras with a bounded approximate identity, Studia Math., 56 (1976), 31-38.

3. W. G. Badé and H. G. Dales, Norms and ideals in radical Banach convolution algebras, J. Functional Analysis, to appear.

4. R. P. Boas, Entire functions, Academic Press, New York, 1964.

5. P. J. Cohen, Factorization in group algebras, Duke Math. J., 26 (1959), 199-206.

6. H. G. Dales, Automatic continuity, a survey, Bull. London Math. Soc., 10 (1978), 129-183.

7. A. Erdelyi, Operational Calculus and Generalized Functions, Holt, Rinehart and Winston, New York, 1962.

8. J. Esterle, Theorems of Gelfand-Mazur type and continuity of epimorphisms from $\mathscr{C}(X)$, J. Functional Analysis.

9. - Universal properties of some commutative radical Banach algebras, J. für die Reine und ang. Math., to appear.

10. - Elements for a classification of commutative radical Banach algebras, preprint.

11. B.E. Johnson, Continuity of centralizers on Banach algebras, J. London Math, Soc., 41 (1966), 639-640.

12. A. M. Sinclair, Factorization, bounded approximate identities and a convolution algebra, J. Functional Analysis, (3) 29 (1978), 308-318. 
13. N. Varopoulos, Continuité des formes linéaires positives sur une algebre de Banach avec involution, C.R. Acad. Sci. Paris, Ser. A 258 (1964), 1121.

Received June 25, 1979 and in revised form May 14, 1980.

Laboratoire Associé aU C.N.R.S.

UNIVERSITÉ DE BORDEAUX I

U.E.R. DE Mathematique et Informatique

351, Cours de la Liberation

33405-TALENCE-(FrancE) 


\section{PACIFIC JOURNAL OF MATHEMATICS}

\section{EDITORS}

DONALD BABBITT (Managing Editor)

University of Galifornia

Los Angeles, California 90024

Hugo RossI

University of Utah

Salt Lake City, UT 84112

C. C. MOORE AND ANDREW OGG

University of California

Berkeley, CA 94720

\section{J. DugundjI}

Department of Mathematics University of Southern California

Los Angeles, California 90007

R. Finn AND J. Milgram

Stanford University

Stanford, California 94305

\section{ASSOCIATE EDITORS}

R. ARENS

E. F. BECKENBACH

B. H. NeUmanN

F. WOLF

K. YosHIDA

\section{SUPPORTING INSTITUTIONS}

UNIVERSITY OF ARIZONA

UNIVERSITY OF BRITISH COLUMBIA

CALIFORNIA INSTITUTE OF TECHNOLOGY

UNIVERSITY OF CALIFORNIA

MONTANA STATE UNIVERSITY

UNIVERSITY OF NEVADA, RENO

NEW MEXICO STATE UNIVERSITY

OREGON STATE UNIVERSITY
UNIVERSITY OF OREGON

UNIVERSITY OF SOUTHERN CALIFONIA

STANFORD UNIVERSITY

UNIVERSITY OF HAWAII

UNIVERSITY OF TOKYO

UNIVERSITY OF UTAH

WASHINGTON STATE UNIVERSITY

UNIVERSITY OF WASHINGTON 


\section{Pacific Journal of Mathematics}

\section{Vol. 94, No. 1 \\ May, 1981}

Willy Brandal, Conditions for being an FGC domain $\ldots \ldots \ldots \ldots \ldots \ldots \ldots$

Allan Calder and Frank Williams, Incompressibility of maps and the

homotopy invariance of Čech cohomology $\ldots \ldots \ldots \ldots \ldots \ldots \ldots \ldots \ldots$

Jacques Chaumat, Quelques propriétés du couple d'espaces vectoriels

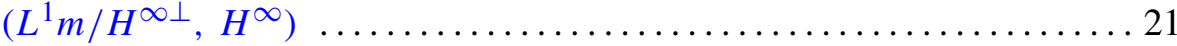

Manfred Droste and Rüdiger Göbel, Products of conjugate permutations . . 47

Jean Esterle, Rates of decrease of sequences of powers in commutative

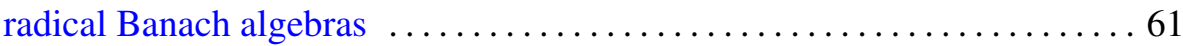

Allan Fryant, Ultraspherical expansions and pseudo analytic functions . . . 83

John Hannah, Homogenization of regular rings of bounded index. II . . . . . 107

Shigeru Haruki, On the theorem of S. Kakutani-M. Nagumo and J. L. Walsh

for the mean value property of harmonic and complex polynomials $\ldots .113$

Hugh M. Hilden, Representations of homology 3-spheres $\ldots \ldots \ldots \ldots \ldots 125$

Craig Huneke, A characterization of locally Macaulay completions . . . . . 131

Takesi Isiwata, Closed ultrafilters and realcompactness ................. 139

Joseph Weston Kitchen, Jr. and David A. Robbins, Tensor products of

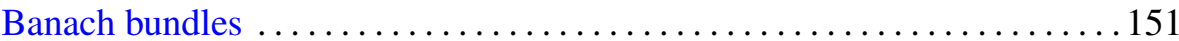

Allan J. Kroopnick, Note on bounded $L^{p}$-solutions of a generalized Liénard

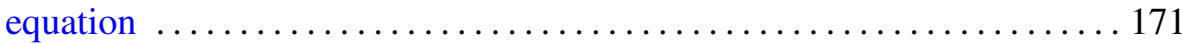

Ajay Kumar and Ajit Kaur Chilana, Spectral synthesis in products and quotients of hypergroups

Charles Livingston, Homology cobordisms of 3-manifolds, knot

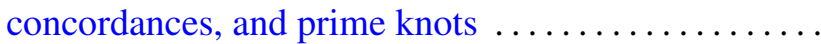

Hans Opolka, Projective representations of finite groups in cyclotomic fields

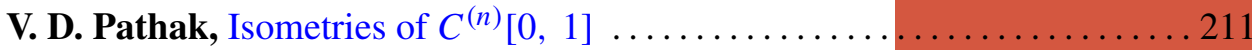

Mark Allan Pinsky, On the spectrum of Cartan-Hadamard manifolds . . . . . 2223

Judith Roitman, The number of automorphisms of an atomic Boolean

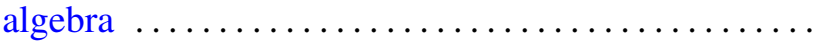

Kai Wang, Locally smooth torus group actions on integral cohomology complex projective spaces 